\title{
Emergency retinal detachment surgery during Covid-19 pandemic: a national survey and local review
}

\author{
Haseeb Akram (1) ${ }^{1} \cdot$ Mohammad Samir Dowlut $^{1} \cdot$ Niral Karia $^{1} \cdot$ Aman Chandra $^{1}$
}

Received: 30 June 2020 / Revised: 10 September 2020 / Accepted: 14 September 2020 / Published online: 22 September 2020

(c) The Royal College of Ophthalmologists 2020

\section{To the Editor:}

During the COVID-19 pandemic, ophthalmology has had the greatest reduction in services compared to most specialties [1]. In response to the article by Wickham et al. [2], we investigated vitreoretinal practice nationwide, using a simple questionnaire. Accompanying this is our own data of retinal detachments.

A 20 question survey was sent to British and Eire Association of Vitreoretinal Surgeons (BEAVRS) members investigating the impact on vitreoretinal surgery during the pandemic. This survey collected data relating to April-May 2020 , which was arguably the peak of the pandemic and major lockdown across the UK.

50 responses were received from separate vitreoretinal units out of 190 questionnaires sent (26\%) to members from an estimated 85 vitreoretinal units in the UK $(59 \%)$ (https://www.eyedocs.co.uk/). The number of RRD presentations were decreased in 66\% (33) respondents compared to normal activity. This is similar to the figure reported by Moorfields Eye Hospital [2]. Trauma remained mostly unchanged in $78 \%$ (39) respondents.

Reassuringly full capacity was available to $62 \%$ (31) of units. The most common reasons for limited access for treatment was a lack of theatre or equipment (Supplementary Fig. 1). Interestingly, for those units with full capacity, $67.7 \%$ still reported a decrease in RRD presentation $(67.7 \%$; Fig. 1). This may be due to the patient anxiety, leading to the macular society encouraging their members to seek

Supplementary information The online version of this article (https:// doi.org/10.1038/s41433-020-01187-1) contains supplementary material, which is available to authorized users.

Haseeb Akram

haseeb.akram5@gmail.com

1 Southend University Hospital Foundation NHS Trust, SouthendOn-Sea, UK

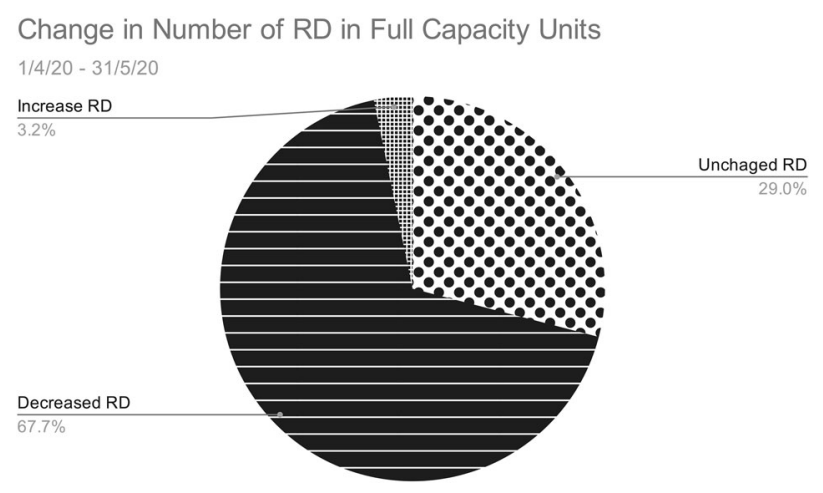

Fig. 1 Change in the number of retinal detachment presentations in units with full capacity: $\boldsymbol{N}=\mathbf{3 1}$. RD retinal detachment; out of 50 respondents, 31 had answered that they were able to provide vitreoretinal treatment at full capacity. Analysis shows $67.7 \%$ of these had decreased number of RD presentations.

ophthalmic service [3]. It does not seem that staff or PPE shortages were a major issue in this survey. Reassuringly, this latter factor agrees with suggestions that institutions have responded well to strong recommendations for PPE, particularly in vitreoretinal surgery [4].

Within our own unit; during the pandemic, we noticed a $292 \%$ increase in RRD cases (total $2020=35$ ) as opposed to the same period in 2019 (total $2019=12$ ). This may be due to more referrals from nearby units who may previously have referred their patients to other centres. There was no statistically significant difference in the proportion of RRD presenting with fovea involvement compared to 2019 (51.4\% vs $66 \%)$. Interestingly, the duration of symptoms preceding presentation to our department for fovea involving RRD during the pandemic (median 3 days, range 1 to 14) decreased significantly compared to 2019 (median 14 days, range $2-42(P=0.006)$. This could be due fewer "pinch points" in the patients' access to vitreoretinal surgeons in our unit; as access to GPs and our emergency services may have been easier and local optometry services severely limited; resulting in patients contacting our department directly. With greater theatre \& surgeon 
availability, due to a reduction in ophthalmic elective work, there may have been an overall positive influence on the patients' surgical journey.

Our own results conflict with the national trend of a reduction in the presentation of RRD. The impact of this nationally may result in significant visual morbidity, which may manifest in the months ahead.

Acknowledgements We would like to thank British and Eire Association of Vitreoretinal Surgeons (BEAVRS) members for participating in our survey.

\section{Compliance with ethical standards}

Conflict of interest The authors declare that they have no conflict of interest.
Publisher's note Springer Nature remains neutral with regard to jurisdictional claims in published maps and institutional affiliations.

\section{References}

1. National Patient and Procedure Volume Tracker. 2020. https://www. stratadecision.com/wp-content/uploads/2020/05/National-Patient-a nd-Procedure-Volume-Tracker-and-Report_May2020.pdf.

2. Wickham L, Hay G, Hamilton R, Wooding J, Tossounis H, da Cruz L, et al. The impact of COVID policies on acute ophthalmology services-experiences from Moorfields Eye Hospital NHS Foundation Trust. Eye (Basingstoke). 2020;34:1-4.

3. Retinal surgeons urge patients not to risk their sight. Macular Society. 2020. https://www.macularsociety.org/news/retinal-surgeons-urge-pa tients-not-risk-their-sight.

4. Chandra A, Haynes R, Burdon M, Laidlaw A, Neffendorf J, Eames I, et al. Personal protective equipment (PPE) for vitreoretinal surgery during COVID-19. Eye. 2020;34:1-4. 